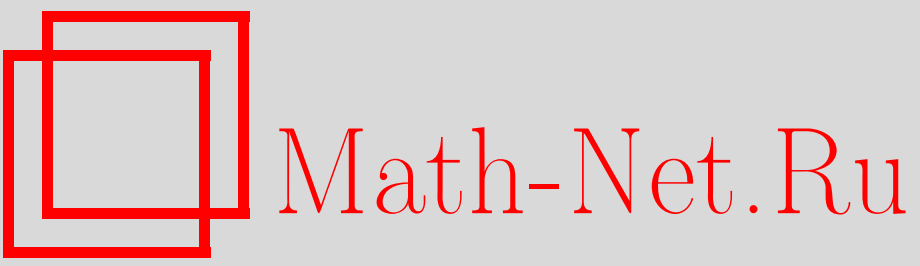

Л. А. Алания, О когомологиях с коэффициентами в локальной системе, близкой к тривиальной, УМН, 1997, том 52, выпуск 2, 151-152

DOI: https://doi.org/10.4213/rm826

Использование Общероссийского математического портала Math-Net.Ru подразумевает, что вы прочитали и согласны с пользовательским соглашением

http://www.mathnet.ru/rus/agreement

Параметры загрузки:

IP: 54.237 .206 .68

26 апреля 2023 г., 18:31:34

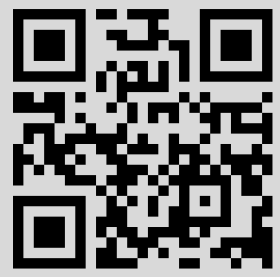




\title{
О КОГОМОЛОГИЯХ С КОЭФФИЦИЕНТАМИ В ЛОКАЛЬНОЙ СИСТЕМЕ, БЛИЗКОЙ К ТРИВИАЛЬНОЙ
}

\author{
Л.А. АлАнИЯ
}

В работе [1] С.П.Новиков поставил вопрос об изучении когомологий “дефформированных” дифференциалов типа $\widetilde{d}=d+t \omega$ в комплексе Де-Рама гладкого многообразия, где $t$ - комплексный параметр, а $\omega$-замкнутая 1-форма. В этой работе была построена спектральная последовательность, которая сходится к когомологиям со значениями в локальной системе $\rho(\gamma)=\exp \int_{\gamma} \omega$,
$\gamma \in H_{1}(M, \mathbb{Z})$.

Были поставлены вопросы об изучении когомологий для многомерных комплексных представлений и для обычных когомологий со значениями в произвольном алгебраически замкнутом поле.

Второй вопрос был успешно решен А.В.Пажитновым в работе [2] для многообразий со свободно абелевой фундаментальной группой. В нашей работе будет исследован первьй из вышеназванных вопросов.

Пусть $M^{n}$ - гладкое компактное многообразие и $\Omega$ - замкнутая матричнозначная 1-форма (матричные элементы $\Omega$ - обычные замкнутые 1-формы).

ОПРЕДЕЛЕниЕ 1 . Последовательность $\Omega=\Omega_{1}, \Omega_{2}, \ldots, \Omega_{k-1}$ 1-форм со значениями в ассоциативной алгебре матриц $M_{k}$ будем назьвать специальной системой $M a с c u$, если выполняются следующие соотношения:

$$
d \Omega_{1}=0, d \Omega_{2}=-\Omega_{1}^{2}, \ldots, d \Omega_{i}=-\sum_{j=1}^{i-1} \Omega_{i-j} \Omega_{j} \text { при } i=1, \ldots, k-1,
$$

умножение форм - внешнее.

Выражение $-\sum_{j=1}^{k-1} \Omega_{k-j} \Omega_{j}$ обозначим через $\langle\Omega\rangle^{k}$ и будем говорить, что специальная степень Масси $\langle\Omega\rangle^{k}=0$, если существует специальная система Масси $\Omega=\Omega_{1}, \Omega_{2}, \ldots, \Omega_{k}$. Выражение $\Omega_{1}, \ldots, \Omega_{k}$ будем назьвать определяющей системой для $\langle\Omega\rangle^{k}$, аналогично Крейнесу [3].

Легко заметить, что условие $(*)$ не зависит от выбора представителя в классе когомологии $\left[\Omega_{1}\right] \in H^{1}\left(\cdot, M_{k}\right)$.

ОПредЕЛЕниЕ 2. Формальная однопараметрическая система матричнозначных 1-форм $\Omega(t)=\Omega_{1} t+\Omega_{2} t+\cdots$ называется формально плоской, если $d \Omega(t)+\Omega(t) \wedge \Omega(t)=0$ как формалшный ряд.

ЛЕмма 1. Если $\Omega(t)$ - формально плоская связность, то все специальные степени Масси ее начального члена $\Omega_{1}$ равны нулю.

ЛЕмма 2. Формально плоскую связность можно проинтегрировать в явном виде, т.е. представить $\Omega(t) \kappa a \kappa ~ g(t)^{-1} d g$, где $g(t)$ - формальный ряд, определенньй на универсальном накрытии многообразия $M^{n}$. Более точно, $g_{i}$ определены на $M_{i}$, где $M_{0}=M^{n} \leftarrow M_{1} \leftarrow M_{2} \leftarrow \cdots$ есть соответствующая центральному ряду группь $\pi_{1}\left(M^{n}\right), \pi_{1} \supset\left[\pi_{1}, \pi_{1}\right] \supset\left[\pi_{1},\left[\pi_{1}, \pi_{1}\right]\right] \supset \cdots$, башня абелевьх накрытий.

Рассмотрим выражение $g(a \cdot x) g^{-1}(x)$, где $a \cdot x$ - сдвиг точки $x$ универсального накрытия на $a \in \pi_{1}(M)$, и разложим его в ряд по $t$. Коэффициенты этого ряда не должны зависеть от $x$. Обозначим их через $I_{1}(a), I_{2}(a), \ldots$ Если для функции на группе ввести оператор дифференцирования $\delta$ следующим образом: $(\delta I)(a, b)=I(a b)-I(a)-I(b)$, легко проверить, что $I_{1}$ задает класс когомологий, а $I_{1}, I_{2}, \ldots, I_{k-1}$ есть определяющая последовательность для $\left\langle I_{1}\right\rangle$.

Работа выполнена при финансовой поддержке Российского фонда фундаментальных исследований (грант № 96-01-01404). 
ОПредЕлЕниЕ 3. Пусть $\Omega$ - замкнутая матричная 1-форма. Будем говорить, что $\langle\Omega\rangle^{\infty}=0$, если существует формально плоское семейство с началшным членом $\Omega$, и коэффицциенты этого ряда будем называть определяющей системой для $\langle\Omega\rangle^{\infty}$.

Аналогично можем определить бесконечные степени Масси и соответствующие определяющие последователшности для групповых 1-коциклов со значениями в матричной алгебре.

ЛЕмма 3. Пусть центральныцй ряд группь $\pi_{1} \supset\left[\pi_{1}, \pi_{1}\right] \supset\left[\pi_{1},\left[\pi_{1}, \pi_{1}\right]\right] \supset \cdots$ обрьввается с некоторого номера $k$ и $I_{1}-$ такой кочикл на группе, что $\left\langle I_{1}\right\rangle^{i}=0$ для всех $i$. Существует определяющая последовательность для $\left\langle I_{1}\right\rangle^{\infty}$ такая, что с некоторого номера $N$ все $I_{k}$ нулевые.

Из этой леммы легко следут следующее

УТВеРЖДЕНИЕ. Если $\Omega_{1}$ - замкнутая матричная 1-форма на гладком многообразии $M^{n},\left\langle\Omega_{1}\right\rangle^{k}=0$ и ряд из леммы 3 для фундаментальной группь стабилизируется, то $\left\langle\Omega_{1}\right\rangle^{\infty}=0$ и, следовательно, существует формально плоское семейство с начальным членом $\Omega_{1}$.

Пусть $\Omega(t)=\Omega_{1} t+\Omega_{2} t+\cdots$ есть формально плоское семейство связностей и $x-$ произвольная замкнутая векторная форма. Если существуют формы $x_{1}, x_{2}, \ldots, x_{n-1}$ такие, что $d x_{i}=\Omega_{i} \wedge x+$ $\Omega_{i-1} \wedge x_{1}+\cdots+\Omega_{1} \wedge x_{i-1}$ для $i \leqslant n$, будем писать, что $\Omega_{n} \wedge x+\Omega_{n-1} \wedge x_{1}+\cdots+\Omega_{1} \wedge x_{n-1} \in$ $\langle x, \Omega\rangle^{n}$. По $\Omega(t)$, следуя работам [1], [2], определим спектральную последовательность $\left(E_{p}^{*}, d_{p}\right)$ следующим образом: $E_{1}^{*}=H^{*}\left(M^{n}, V\right), d_{1}=\Omega_{1} \wedge \cdot, d_{p}(x)=\langle x, \Omega\rangle^{p}$. Корректность этого определения легко проверяется.

ТЕОРемА. Пусть для многообразия $M^{n}$ чентральный ряд группь $\pi_{1}\left(M^{n}\right)$ имеет конечную длину $k$ и $\Omega_{1}-$ такая 1-форма, что $\left\langle\Omega_{1}\right\rangle^{k}=0$. В этих предположениях существует однопараметрическое семейство представлений $g(t): \pi_{1}\left(M^{n}\right) \rightarrow G L(k), u$ спектральная последовательность, построенная вышеописанным способом по $g^{-1} d g$, сходится к когомологиям многообразия $M^{n}$ с коэффициентами в локальной системе $g(t)$ для почти всех значений $t$.

\section{СПИСОК ЛИТЕРАТУРЫ}

[1] Новиков С.П. // Докл. АН. 1986. Т. 287. № 6. С. 1321-1324. [2] Пажитнов А.В. // Докл. АН. 1988. Т. 300. №6. C. 1316-1320. [3] Kraines D. // Trans. Amer. Math. Soc. 1966. V. 124. № 3. P. 431-449.

Московский государственньй

Принято редколлегией университет им. М.В.Ломоносова 